OPEN ACCESS

Edited by:

Ke Chen,

Nanjing University, China

Reviewed by:

Weiren Zhu,

Shanghai Jiao Tong University, China Wang Shen-Yun, Nanjing University of Information Science and Technology, China

${ }^{*}$ Correspondence:

Liansheng Wang wlswls1982@126.com

Specialty section:

This article was submitted to

Metamaterials,

a section of the journal

Frontiers in Materials

Received: 23 June 2021 Accepted: 16 July 2021

Published: 26 July 2021

Citation:

Wang L, Xia D, Fu Q, Ding $X$ and Wang Y (2021) A Switchable UltraWideband Metamaterial Absorber with Polarization-Insensitivity and Wide-

incident Angle at $\mathrm{THz}$ Band.

Front. Mater. 8:729495

doi: 10.3389/fmats.2021.729495

\section{A Switchable Ultra-Wideband Metamaterial Absorber with Polarization-Insensitivity and Wide-incident Angle at THz Band}

\author{
Liansheng Wang ${ }^{1 *}$, Dongyan Xia ${ }^{2}$, Quanhong $\mathrm{Fu}^{3}$, Xueyong Ding ${ }^{1}$ and Yuan Wang ${ }^{1}$ \\ ${ }^{1}$ Science and Technology Department, Sanya University, Sanya, China, ${ }^{2}$ Finance and Economics Department, Sanya University, \\ Sanya, China, ${ }^{3}$ Science Department, Northwestern Polytechnical University Xi'an, Shanxi, China
}

In this paper, we report a switchable ultra-wideband metamaterial absorber with polarization-insensitivity and wide-incident angle at $\mathrm{THz}$ band which is composed of $\mathrm{VO}_{2}$ disk, polyimide dielectric substrate, and gold ground plane. The results show that the absorption is greater than $90 \%$ from $3.5-8 \mathrm{THz}$ for a temperature of $300 \mathrm{~K}$ and this absorption band disappears when the temperature rises to $350 \mathrm{~K}$. The absorption property of our proposed metamaterial absorber is insensitive to polarization states and angles and it can withhold high absorption of more than $80 \%$ for wide-incident angles, up to $60^{\circ}$ for TE mode and TM mode. The wideband absorption mechanism is elucidated using an effective medium and surface current analysis.

Keywords: ultra-wideband, metamaterial absorber, wide incident angle, $\mathrm{THz}$ band, switchable

\section{INTRODUCTION}

Absorbing materials refer to natural materials or metamaterials constructed by electromagnetic structures that can absorb electromagnetic waves in free space. Traditional absorbing material is a kind of absorbing material coated on the target surface, which is usually made of ferrite, carbon powder, and other absorbents mixed with some non-metallic substrates such as thermoplastic or epoxy resin. The traditional absorbing material usually has the disadvantage of having a narrow absorption band, small absorption angle and being bulky. In 2008, Landy et al. (Landy et al., 2008) firstly proposed the concept of metamaterial absorber. Since then, metamaterial absorber has attracted wide attention. Many researchers have achieved excellent results on wideband absorption, polarization-insensitivity, tunable absorption, and so on from microwave to the visible light band (Chen, 2012; Wang et al., 2014a;; Hao et al., 2014; Chen et al., 2015; Lin et al., 2016; Chen et al., 2019; Xie et al., 2019; Zhang et al., 2019; Zheng et al., 2019; Quader et al., 2020; Zhang et al., 2021). At present, the researches of metamaterial absorber are flourishing to achieve wideband absorption, polarization-insensitive absorption, tunable absorption, and multi-band absorption (Aydin et al., 2011; Li et al., 2011; Ding et al., 2012; Xu et al., 2012; Argyropoulos et al., 2013). However, the narrow-incident angle of metamaterial absorbers limit their applications in practice. Consequently, it is necessary to design metamaterial absorbers with polarizationinsensitivity and wide-incident angle.

Many methods have been devoted to widen the incident angle of metamaterial absorbers. In 2017, Fan J X et al. proposed a wide-angle wideband terahertz metamaterial absorber with a multilayered heterostructure (Fan et al., 2017). In 2018, Huang X T et al. designed a wide-angle perfect metamaterial absorber based on cave-rings and the complementary patterns (Huang et al., 2018a), its absorption is 

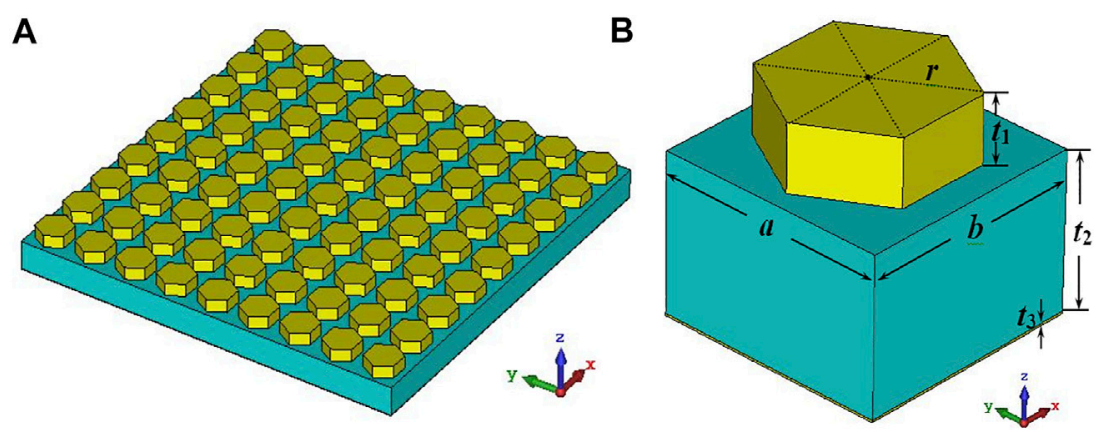

FIGURE 1 | Schematic diagram of the metamaterial absorber unit cell, (A) $9 \times 9$ array of the unit cell, (B) perspective view of the unit cell.

over $92 \%$ at around $6.53 \mathrm{THz}$ when the incident angle for the TE mode is up to $80^{\circ}$, and at TM mode, its absorption at $7.64 \mathrm{THz}$ is greater than $92 \%$ even for an incident angle of up to $70^{\circ}$. In the same year, Huang X T designed multiband ultrathin polarizationinsensitive terahertz perfect absorber (Huang et al., 2018b), it presented the ability to maintain high absorption of more than $80 \%$ for a large incident angle up to $60^{\circ}$ for both TE and TM modes.

However, the absorption band of the above mentioned wideangle terahertz metamaterial absorber is narrow, this limits their application in practice. In this work, we propose a switchable ultra-wideband metamaterial absorber with polarizationinsensitivity and wide-incident angle at the $\mathrm{THz}$ band. Its absorption is over $90 \%$ from $3.5-8 \mathrm{THz}$ for a temperature of $300 \mathrm{~K}$ and the absorption band fades away when the temperature rises to $350 \mathrm{~K}$. It remains highly absorptive with over $80 \%$ absorption for a wide-incident angle up to at $60^{\circ}$ for both TE mode and TM mode. Compared with the reported wide-angle terahertz wideband metamaterial absorber (He et al., 2011; Wang et al., 2014b; Li et al., 2015; Huang et al., 2018c; Dinh et al., 2021), our designed metamaterial absorber has the advantages of wider absorption bandwidth and incident angle.

\section{MODEL DESIGN}

As illustrated in Figure 1, the unit cell of our proposed metamaterial absorber consists of $\mathrm{VO}_{2}$ disk, polyimide dielectric substrate, and gold ground plane. The periodicities of the unit cell are $a=b=12 \mu \mathrm{m}$. The geometrical parameter of the $\mathrm{VO}_{2}$ disk is $r=5 \mu \mathrm{m}$. During the simulation process, the $\mathrm{VO}_{2}$ disk is set as a thermally tunable resistance film material with the conductivity $\sigma=2 \times 10^{2} \mathrm{~S} / \mathrm{m}$ when the temperature $T=300 \mathrm{~K}$ and $\sigma=2 \times 10^{5} \mathrm{~S} / \mathrm{m}$ when temperature $T=350 \mathrm{~K}$ according to the reference (Dao et al., 2019), the thickness of $\mathrm{VO}_{2}$ disk is $3 \mu \mathrm{m}$. The polyimide dielectric substrate selected has a relative dielectric constant of $\varepsilon_{r}=2.35$, a loss of $\tan \delta=2.35$ and its thickness is $7 \mu \mathrm{m}$. The thickness of the gold ground plane $\left(\sigma=4.56 \times 10^{7} \mathrm{~S} / \mathrm{m}\right)$ is $0.1 \mu \mathrm{m}$. The difficulty of the realization of our proposed metamaterial absorber in practice is the preparation of $\mathrm{VO}_{2}$ disk.

The full-wave electromagnetic simulation of our proposed metamaterial absorber is performed with CST Microwave Studio.

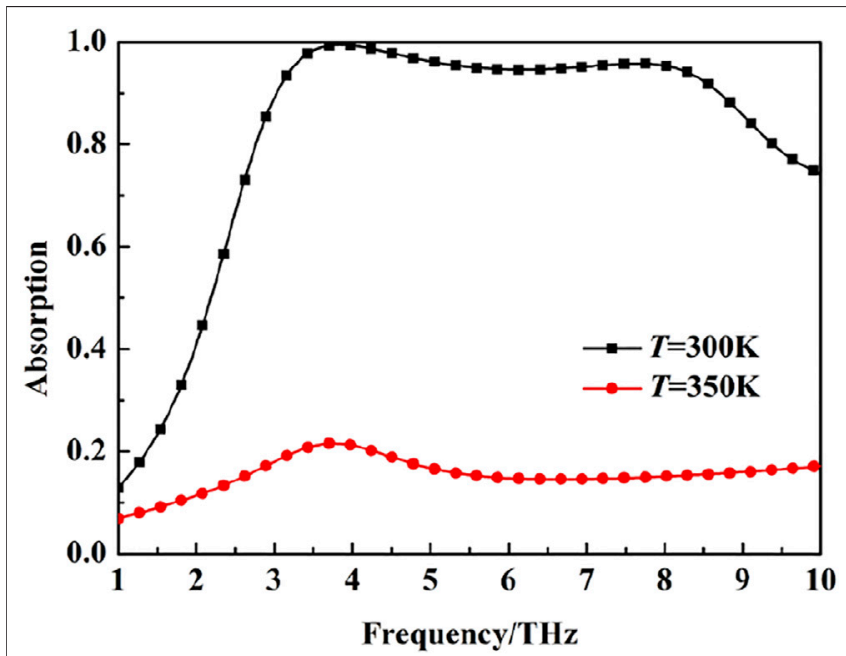

FIGURE 2 | The absorption of the metamaterial absorber under the temperatures $T=300 \mathrm{~K}$ and $T=350 \mathrm{~K}$

Throughout the simulation process, the boundary conditions of $x$ and $y$ directions are set as unit cell, the $z$-direction is set as open. All + Floquet ports are used to simulate the incoming and outgoing waves. The electromagnetic parameters are calculated using a frequency-domain electromagnetic solver.

\section{RESULTS AND DISCUSSION}

For the metamaterial absorber, the absorption can be calculated by $A(\omega)=1-\left|S_{11}\right|^{2}-\left|S_{21}\right|^{2}\left(S_{11}\right.$ and $S_{21}$ are the reflection and transmission). For our designed metamaterial absorber, there is no transmission due to the gold ground plane. Thus, the expression of absorption can be simplified as $A(\omega)=1-\left|S_{11}\right|^{2}$. The simulated absorptions of the metamaterial absorber with the temperature $T=300 \mathrm{~K}$ and $T=350 \mathrm{~K}$ are shown in Figure 2. The absorption is more than $90 \%$ from $3.5-8 \mathrm{THz}$ for a temperature of $300 \mathrm{~K}$ and the absorption band disappears when the temperature rises to $350 \mathrm{~K}$.

To understand the absorption mechanism, the normalized input impedance of the metamaterial absorber with the free space 


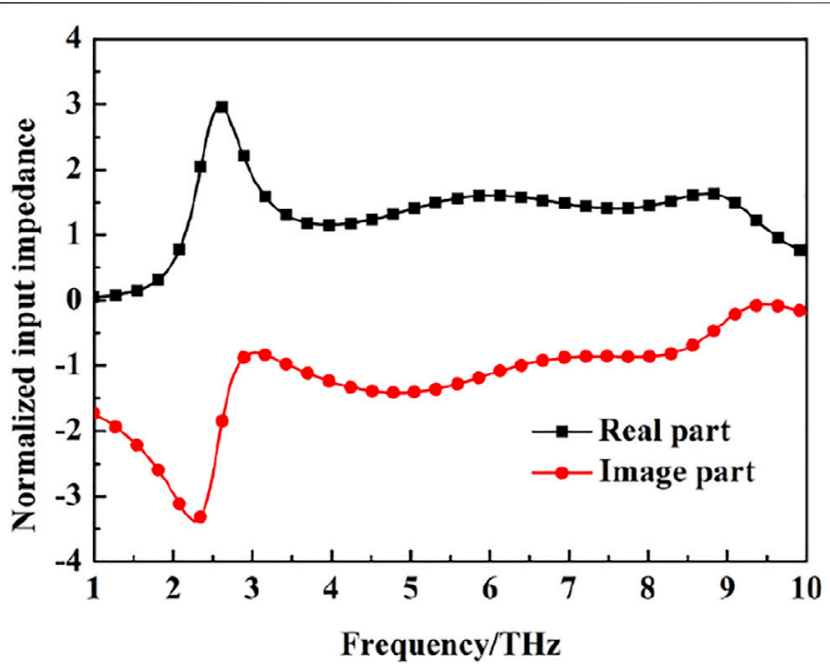

FIGURE 3 | The normalized input impedance of the metamaterial absorber with free space $(T=300 \mathrm{~K})$.

for normal incidence $(T=300 \mathrm{~K})$ is retrieved from the simulated $S_{11}$ and $S_{21}$ parameters by using the scattering parameter method (Smith and Schultz, 2002), as shown in Figure 3. The real part of the normalized input impedance of the metamaterial absorber with free space is nearly unity from $3.5-8 \mathrm{THz}$, which indicates that our proposed metamaterial absorber acquires an impedance match with free space from $3.5-8 \mathrm{THz}$, which means that the reflection is nearly zero. As an outcome, the absorption will be very high.

The wideband absorption mechanism of the proposed metamaterial absorber is further clarified in Figure 4. Figure 4 shows the surface current of the metamaterial absorber on the $\mathrm{VO}_{2}$ disk and gold ground plane at $6 \mathrm{THz}(T=300 \mathrm{~K})$. It can be seen that the induced anti-parallel currents on these two layers prove that magnetic resonance is formed at $6 \mathrm{THz}$. Therefore, the absorption of the metamaterial absorber at $6 \mathrm{THz}$ originates from the magnetic resonance (Son et al., 2014). The reason for wideband absorption is that the circuit resonant structure formed by $\mathrm{VO}_{2}$ disk, polyimide dielectric substrate, and a gold ground plane can realize the impedance match between the metamaterial absorber and free space over a wide frequency range near the resonant frequency, and can then broaden the absorption band (Costa et al., 2010; Zhang et al., 2013).

The power loss density distributions at different frequencies are monitored at $T=300 \mathrm{~K}$, as shown in Figure 5. It can be observed that there are similar power loss density distributions at different frequencies at 4 and $6 \mathrm{THz}$, the power losses all concentrate on the front part of polyimide dielectric substrates.

Figure 6 shows the absorption of the metamaterial absorber at different polarization angles $(T=300 \mathrm{~K})$. Owing to the rotational symmetry of the unit cell, the absorption under different polarization angles is the same.

The waves are usually incident on to metamaterial absorber with different incident angles. Figure 7 shows the absorption of the metamaterial absorber with different incident angles at TE and TM mode $(T=300 \mathrm{~K})$. For TE and TM mode, the absorption is over $80 \%$ for incident angles below $60^{\circ}$ from $3.5-8 \mathrm{THz}$. However, the absorption decreases noticeably for incident angles beyond $60^{\circ}$. This indicates that the absorption property of the metamaterial absorber has the advantage of being responsive towards wide-incident angle.

Figure 8 and Figure $\mathbf{9}$ shows the absorption of the metamaterial absorber for different thickness of $\mathrm{VO}_{2}$ disk and polyimide dielectric substrate $(T=300 \mathrm{~K})$. The absorption band of the metamaterial absorber gradually shifts to a higher frequency with the increase of thickness of $\mathrm{VO}_{2}$ disk. The absorption of the metamaterial absorber gradually decreases with the increase of the thickness of the polyimide dielectric substrate.

\section{CONCLUSION}

In conclusion, we propose a switchable ultra-wideband terahertz metamaterial absorber with polarization-insensitivity and wideincident angle. It is composed of a $\mathrm{VO}_{2}$ disk, polyimide dielectric
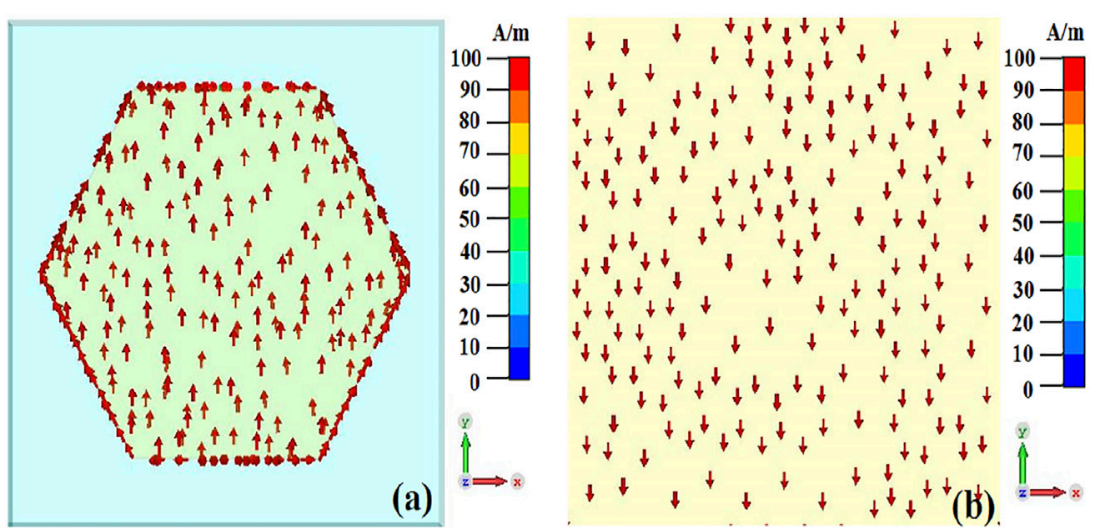

FIGURE 4 | The surface current of the metamaterial absorber at $6 \mathrm{THz}(T=300 \mathrm{~K})$, (A) $\mathrm{VO}_{2}$ disk; (B) gold ground 

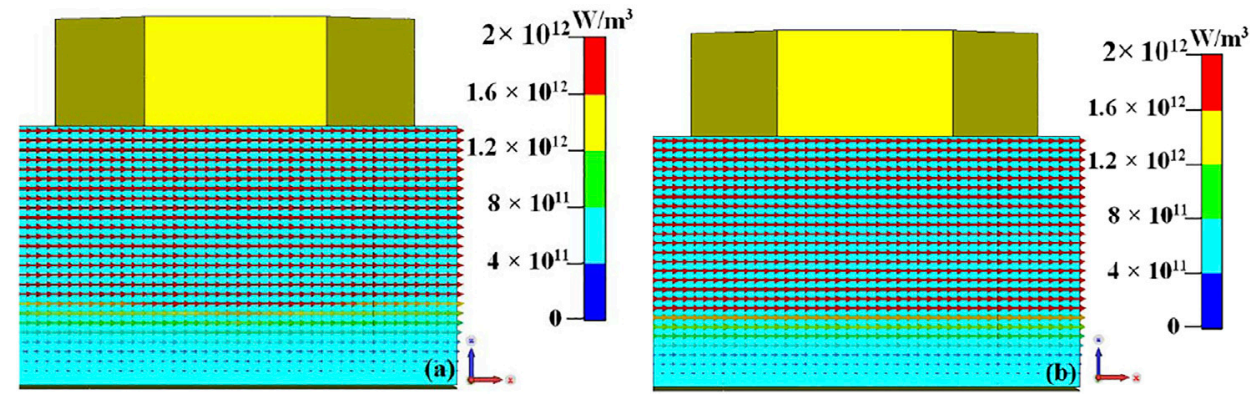

(b)

FIGURE 5 | The power loss density distribution of the metamaterial absorber $(T=300 \mathrm{~K}),(\mathbf{A}) 4 \mathrm{THz}$, (B) $6 \mathrm{THz}$.

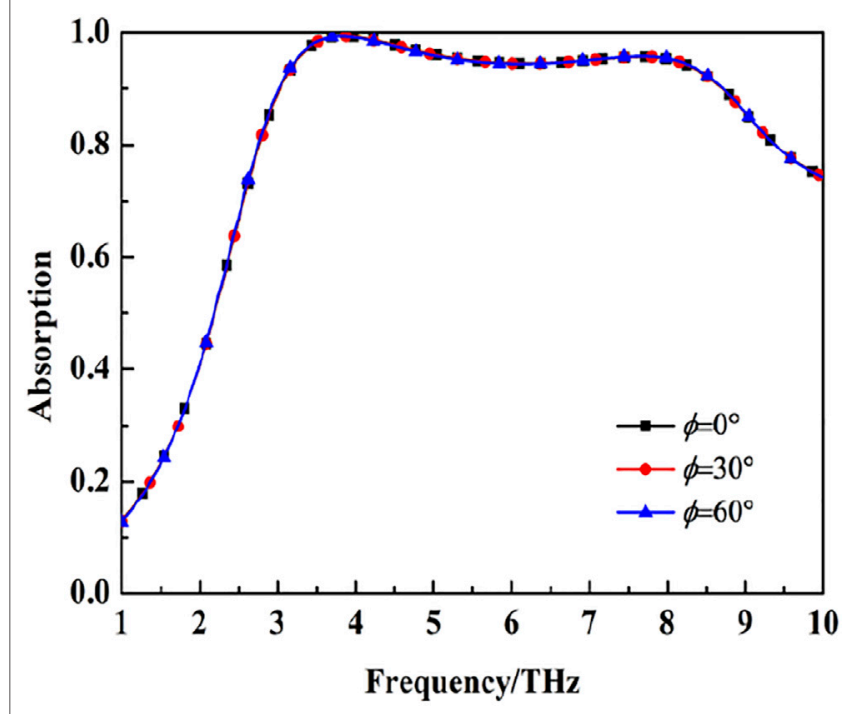

FIGURE 6 | The absorption of the metamaterial absorber at different polarization angles $(T=300 \mathrm{~K})$.

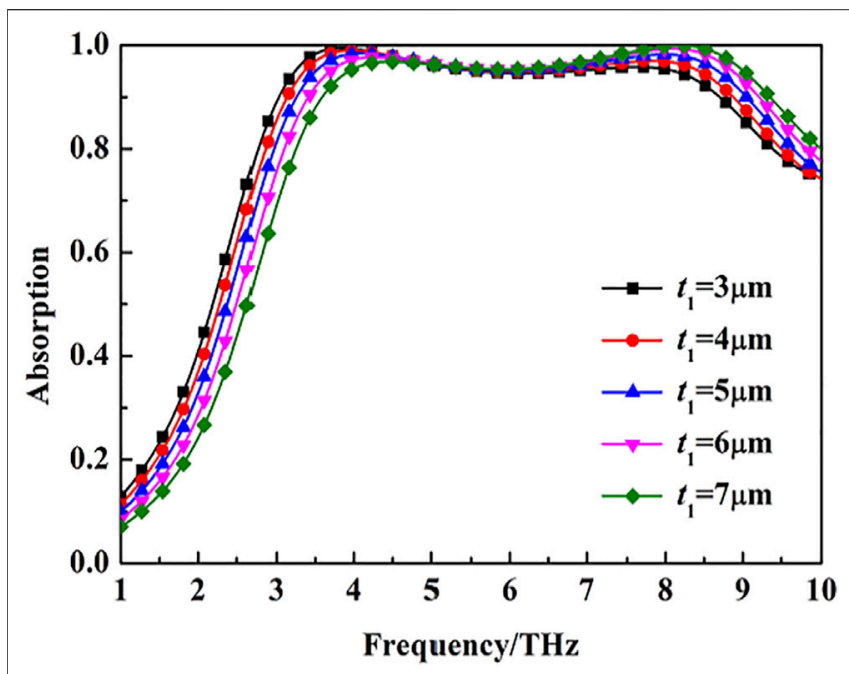

FIGURE 8 | The absorption of the metamaterial absorber with different thickness of $\mathrm{VO}_{2}$ disk $(T=300 \mathrm{~K})$.
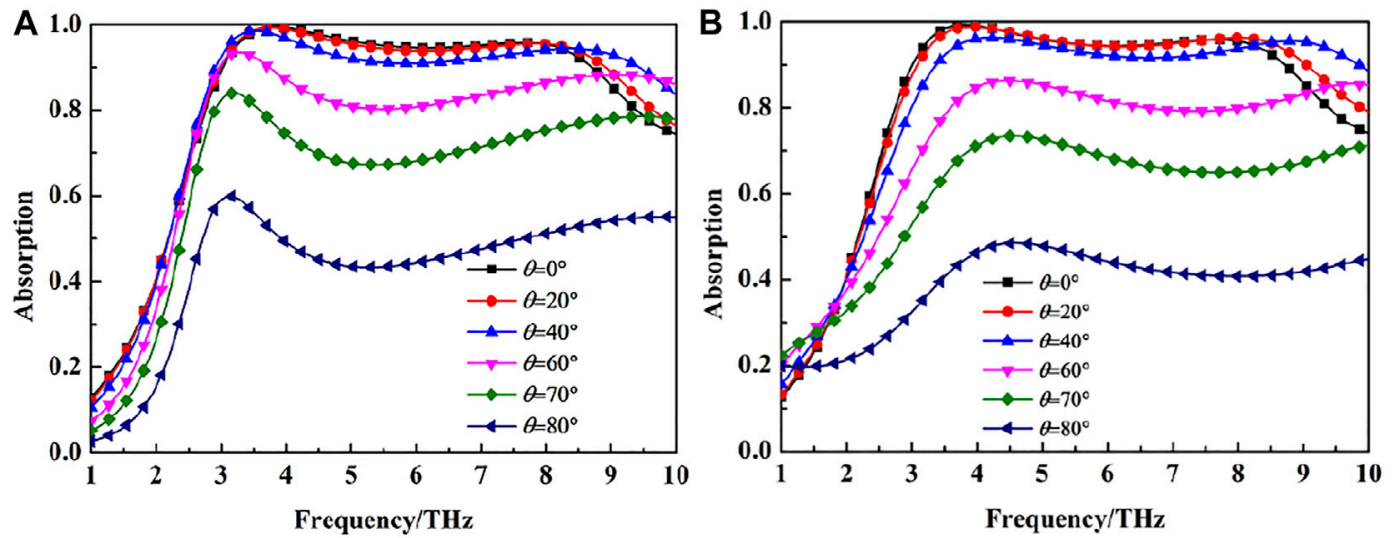

FIGURE 7 | The absorption of the metamaterial absorber at different incident angles (T=300 K), (A) TE mode, (B) TM mode. 


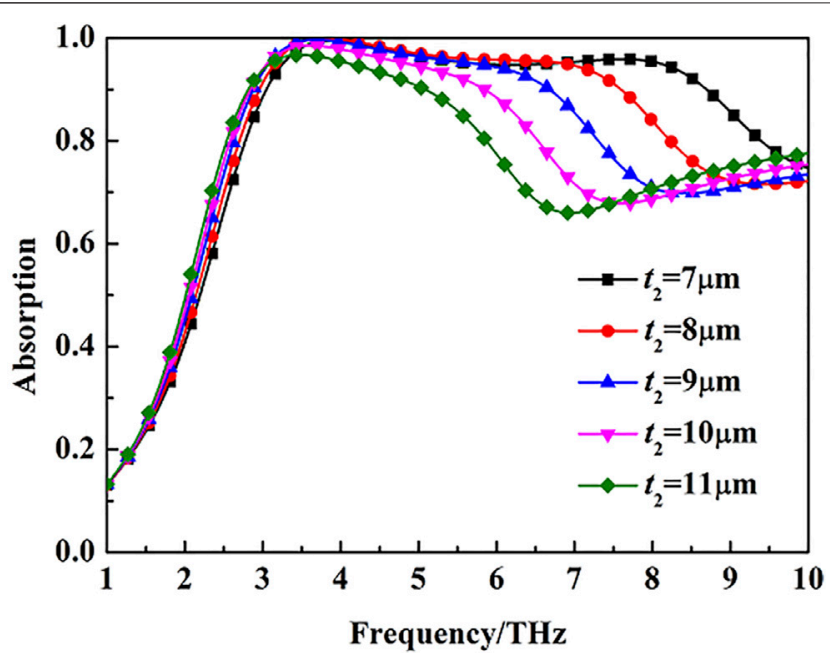

FIGURE 9 | The absorption of the metamaterial absorber with different thickness of polyimide dielectric substrates $(T=300 \mathrm{~K})$.

substrate, and gold ground plane. The simulation results show that the absorber provides a strong wideband absorption for incident

\section{REFERENCES}

Argyropoulos, C., Le, K. Q., and Mattiucci, N. (2013). Broadband absorbers and selective emitters based on plasmonic Brewster metasurfaces. Phys. Rev. B. 87, 205112. doi:10.1103/physrevb.87.205112

Aydin, K., Ferry, V. E., Briggs, R. M., and Atwater, H. A. (2011). Broadband polarization-independent resonant light absorption using ultrathin plasmonic super absorbers. Nat. Commun. 2, 517. doi:10.1038/ncomms 1528

Chen, H. T. (2012). Interference theory of metamaterial perfect absorbers. Opt. Express. 20, 7165-7172. doi:10.1364/oe.20.007165

Chen, K., Jia, N., Sima, B., Zhu, B., Zhao, J., and Feng, Y. (2015). Microwave absorber based on permeability-near-zero metamaterial made of Swiss roll structures. J. Phys. D: Appl. Phys. 48, 455304. doi:10.1088/0022-3727/48/45/ 455304

Chen, K., Luo, X., Ding, G., Zhao, J., Feng, Y., and Jiang, T. (2019). Broadband microwave metamaterial absorber with lumped resistor loading. EPJ Appl. Metamat. 6, 1. doi:10.1051/epjam/2018011

Costa, F., Monorchio, A., and Manara, G. (2010). Analysis and design of ultra-thin electromagnetic absorbers comprising resistively loaded high impedance surfaces. IEEE Trans. Antennas Propag. 58 (5), 1551-1558. doi:10.1109/ tap.2010.2044329

Dao, R. N., Kong, X. R., Zhang, H. F., and Su, X. R. (2019). A tunable broadband terahertz metamaterial absorber based on the vanadium dioxide. Optik 180, 619-625. doi:10.1016/j.ijleo.2018.12.004

Ding, F., Cui, Y. X., Ge, X. C., Jin, Y., and He, S. L. (2012). Ultra-broadband microwave metamaterial absorber. Appl. Phys. Lett. 100, 103506. doi:10.1063/ 1.3692178

Dinh, M. Q., Huynh, T. V., Khuyen, B. X., Tung, B. S., Lam, V. D., Ngo, S. T., et al. (2021). Graphene-integrated hybridized metamaterials for wide-angle tunable $\mathrm{THz}$ absorbers. Photonic. nanostruct. 45, 100924. doi:10.1016/ j.photonics.2021.100924

Fan, J., Xiao, D., Wang, Q., Liu, Q., and Ouyang, Z. (2017). Wide-angle broadband terahertz metamaterial absorber with a multilayered heterostructure. Appl. Opt. 56, 4388-4391. doi:10.1364/ao.56.004388

Hao, J., Lheurette, É., Burgnies, L., Okada, É., and Lippens, D. (2014). Bandwidth enhancement in disordered metamaterial absorbers. Appl. Phys. Lett. 105, 081102. doi:10.1063/1.4894181 waves from $3.5-8 \mathrm{THz}$ for a temperature of $300 \mathrm{~K}$ and this strong absorption band diminishes when the temperature rises to $350 \mathrm{~K}$. We also show that the absorption property of our proposed metamaterial absorber is insensitive to polarization states and angles and it responses well under wide-incident angles as well.

\section{DATA AVAILABILITY STATEMENT}

The raw data supporting the conclusion of this article will be made available by the authors, without undue reservation.

\section{AUTHOR CONTRIBUTIONS}

The contribution of QF is analysising the model. The contribution of DX, YW and $\mathrm{XD}$ is data processing.

\section{FUNDING}

This work is supported by Hainan Provincial Natural Science Foundation of China (Granted number: 620MS062).

He, X. J., Wang, Y., Wang, J. M., Gui, T. L., and Wu, Q. (2011). Dual-band terahertz metamaterial absorber with polarization insensitivity and wide incident angle. Prog.electromagn. res. 115, 381-397. doi:10.2528/pier11022307

Huang, M. L., Cheng, Y. Z., Cheng, Z. Z., Chen, H. R., Mao, X. S., and Gong, R. Z. (2018). Based on graphene tunable dual-band terahertz metamaterial absorber with wide-angle. Opt.commun. 415 (1), 194-201. doi:10.1016/ j.optcom.2018.01.051

Huang, X., Lu, C., Rong, C., and Liu, M. (2018). Wide-angle perfect metamaterial absorbers based on cave-rings and the complementary patterns. Opt. Mater. Express. 8, 2520-2531. doi:10.1364/ome.8.002520

Huang, X. T., Lu, C. H., Rong, C. C., Hu, Z. Y., and Liu, M. H. (2018). Multiband ultrathin polarization-insensitive terahertz perfect absorbers with complementary metamaterial and resonator based on high-order electric and magnetic resonances. IEEE Photonics. J. 10 (6), 4600811. doi:10.1109/ jphot.2018.2878455

Landy, N. I., Sajuyigbe, S., Mock, J. J., Smith, D. R., and Padilla, W. J. (2008). Perfect metamaterial absorber. Phys. Rev. Lett. 100, 207402. doi:10.1103/ physrevlett.100.207402

Li, L., Yang, Y., and Liang, C. H. (2011). A wide-angle polarization-insensitive ultra-thin metamaterial absorber with three resonant modes. J. Appl. Phys. 110, 063702. doi:10.1063/1.3638118

Li, X. W., Liu, H. J., Sun, Q. B., and Huang, N. (2015). Ultra-broadband and polarization-insensitive wide-angle terahertz metamaterial absorber. Photonic. nanostruct. 15, 81-88. doi:10.1016/j.photonics.2015.04.002

Lin, X. Q., Mei, P., Zhang, P. C., Chen, Z. Z. D., and Fan, Y. (2016). Development of a resistor-loaded ultrawideband absorber with antenna reciprocity. IEEE Trans. Antennas Propagat. 64, 4910-4913. doi:10.1109/tap.2016.2598202

Quader, S., Akram, M. R., Xiao, F. J., and Zhu, W. R. (2020). Graphene based ultrabroadband terahertz metamaterial absorber with dual-band tunability. J. Opt. 22, 095104. doi:10.1088/2040-8986/aba814

Smith, D. R., and Schultz, S. (2002). Determination of negative permittivity and permeability of metamaterials from reflection and transmission coefficients. Phys. Rev. B 65, 195104. doi:10.1103/physrevb.65.195104

Son, T. B., Van, K. B., Van, D. N., Young, J. Y., Ki, W. K., Dinh, L. V., et al. (2014) Small-size metamaterial perfect absorber operating at low frequency. Adv. Nat. Sci. Nanosci. Nanotechnol. 5, 045008. doi:10.1088/2043-6262/5/4/045008

Wang, B.-X., Wang, L.-L., Wang, G.-Z., Huang, W.-Q., Li, X.-F., and Zhai, X. (2014). Theoretical investigation of broadband and wide-angle terahertz 
metamaterial absorber. IEEE Photon. Technol. Lett. 26 (2), 111-114. doi:10.1109/lpt.2013.2289299

Wang, B. X., Wang, L. L., Wang, G. Z., Huang, W. Q., and Zhai, X. (2014). Broadband, polarization-insensitive and wide-angle terahertz metamaterial absorber. Phys. Scripta 89 (11), 115501. doi:10.1088/0031-8949/89/11/115501

Xie, J. W., Quader, S., Xiao, F. J., He, C., Liang, X. L., Zhu, R. W., et al. (2019). Truly all-dielectric ultra-broadband metamaterial absorber: Water-based and ground-free. IEEE Trans. Antennas Propag. 18 (3), 536-540. doi:10.1109/ lawp.2019.2896166

Xu, H. X., Wang, G. M., Qi, M. Q., Liang, J. G., Gong, J. Q., and Xu, Z. M. (2012). Triple-band polarization-insensitive wide-angle ultra-miniature metamaterial transmission line absorber. Phys. Rev. B 86, 205104. doi:10.1103/ physrevb.86.205104

Zhang, H. B., Zhou, P. H., Lu, H. P., Xu, Y. Q., Liang, D. F., and Deng, L. J. (2013). Resistance selection of high impedance surface absorbers for perfect and broadband absorption. IEEE Trans. Antennas Propag. 61 (2), 976-979. doi:10.1109/tap.2012.2226225

Zhang, J., Wei, X. Z., Premaratne, M., and Zhu, W. R. (2019). Experimental demonstration of electrically tunable broadband coherent perfect absorber based on graphene-electrolyte-graphene sandwich structure. Photon. Res. 7 (8), 868-874. doi:10.1364/prj.7.000868

Zhang, J. Z., Li, Z. F., Shao, L. D., and Zhu, W. R. (2021). Dynamical absorption manipulation in a graphene-based optically transparent and flexible metasurface. Carbon 176, 374-382. doi:10.1016/ j.carbon.2021.01.137

Zheng, Y., Chen, K., Jiang, T., Zhao, J., and Feng, Y. (2019). Multi-octave microwave absorption via conformal metamaterial absorber with optical transparency. J. Phys. D: Appl. Phys. 52, 335101. doi:10.1088/1361-6463/ab2365

Conflict of Interest: The authors declare that the research was conducted in the absence of any commercial or financial relationships that could be construed as a potential conflict of interest.

Publisher's Note: All claims expressed in this article are solely those of the authors and do not necessarily represent those of their affiliated organizations, or those of the publisher, the editors and the reviewers. Any product that may be evaluated in this article, or claim that may be made by its manufacturer, is not guaranteed or endorsed by the publisher.

Copyright (c) 2021 Wang, Xia, Fu, Ding and Wang. This is an open-access article distributed under the terms of the Creative Commons Attribution License (CC BY). The use, distribution or reproduction in other forums is permitted, provided the original author(s) and the copyright owner(s) are credited and that the original publication in this journal is cited, in accordance with accepted academic practice. No use, distribution or reproduction is permitted which does not comply with these terms. 